\title{
Labyrinthe
}

22 | 2005 (3)

La Biopolitique (d')après Michel Foucault

\section{« Dispositifs de sécurité » et épidémie de sida}

\section{Delphine Moreau}

\section{(2) OpenEdition}

\section{Journals}

Édition électronique

URL : http://journals.openedition.org/labyrinthe/1038

DOI : $10.4000 /$ labyrinthe.1038

ISSN : 1950-6031

Éditeur

Hermann

Édition imprimée

Date de publication : 1 novembre 2005

Pagination : 101-110

Référence électronique

Delphine Moreau, « «Dispositifs de sécurité » et épidémie de sida », Labyrinthe [En ligne], 22 | 2005 (3) mis en ligne le 22 juillet 2008, consulté le 30 avril 2019. URL : http://journals.openedition.org/ labyrinthe/1038; DOI : 10.4000/labyrinthe.1038 


\title{
«DISPOSITIFS DE SÉCURITÉ » ET ÉPIDÉMIE DE SIDA
}

\author{
Delphine MOREAU \\ delphmoreau@free.fr
}

L'épidémie de sida à son apparition avait tout pour faire renaître les peurs archaïques des grandes épidémies (peste, lèpre, syphilis) et avec elles les moyens les plus défensifs et répressifs de s'en prémunir: identifiée en premier lieu au sein de populations déjà stigmatisées (homosexuels, toxicomanes, prostituées, étrangers...), très vite associée à une transmission par le sexe et le sang, auxquels s'ajoutaient les angoisses de contamination par tout contact, elle aurait pu donner lieu à une réponse brutale, associant dépistage obligatoire (une fois le virus identifié et le test mis au point en 1985), isolement des séropositifs, interdiction de leur circulation, voire enfermement, surveillance de ce que l"on a appelé les « groupes à risque », associant ce que Foucault nommait « dispositifs juridico-légaux » et « techniques disciplinaires ». Sous différentes formes et à différents degrés, c'est le choix qui a pu être fait dans certains pays. Mais ce n'est pas le cas en France.

Pour caractériser les choses rapidement, on pourrait dire qu'il n'y a eu :

- ni politique brutale de séparation, de mise à l'écart des malades, énonciation d'interdits (sur un plan légal, moral ou religieux : interdit de l'homosexualité, de l'infidélité ou du multipartenariat, voire de la sexualité), et mise en place de dispositifs répressifs (répression de l'usage de drogues, pénalisation de la transmission, enfermement des séropositifs), selon le modèle que Foucault qualifierait de juridico-légal,

- ni système de quadrillage, de fichage des personnes séropositives, avec dépistage obligatoire, et de surveillance, avec par exemple recherche des partenaires sexuels, sur un modèle disciplinaire, comme cela a pu être fait en Suède ${ }^{1}$.

Mais au contraire, c'est le principe du dépistage anonyme et gratuit qui a été adopté, de campagnes d'information et de prévention adressées 
à différents types de publics, la vente libre des seringues puis un système d'échange de seringues, et d'un important soutien à la recherche médicale pour trouver traitements et techniques prophylactiques. La déclaration des nouveaux cas de sida avérés puis des cas de séropositivité a été rendue obligatoire, mais leur anonymat garanti.

C'est le choix de la responsabilisation des acteurs eux-mêmes pour leur propre protection et celle des autres qui a été fait - ce qu'on a appelé le principe de la « responsabilité partagée »-, d'un effort pour faire entrer dans les pratiques individuelles les moyens de juguler l'épidémie. Il ne s'agissait pas seulement d'un choix «éthique » ou politique, d'un refus de limiter les libertés des personnes, mais aussi d'un choix stratégique misant sur une plus grande compliance des individus, leur plus grande adhésion à des conduites de protection, partant de l'hypothèse d'une lutte contre l'épidémie plus efficace si les démarches venaient d'une conduite volontaire plutôt que d'une contrainte externe.

Comment décrire ce nouvel agencement du pouvoir et du savoir face à une épidémie? Le nouveau modèle de technologie de pouvoir que Foucault distingue dans $S T P^{2}$ des normes juridico-légales et des disciplines, qu'il nomme dispositifs de sécurité ou biopouvoir, nous a semblé d'une certaine pertinence. On pourrait même avancer l'hypothèse selon laquelle, plus qu'un exemple, l'épidémie de sida constitue l'épidémieparadigme de ce nouveau type de technologie de pouvoir, après que l'exclusion des lépreux eut été celui du modèle juridico-légal (séparation, définition de l'interdit et de l'exclu), et les règlements de peste, celui des techniques disciplinaires de quadrillage, d'individualisation et de surveillance ${ }^{3}$. Nous en resterons à ce stade à des pistes de réflexion, aux indications des limites et des problèmes que l'application de ce modèle du biopolitique à cet exemple pose, d'autant qu'il est resté à l'état d'ébauche, son étude étant sans cesse reportée ${ }^{4}$.

\footnotetext{
2. Voir également la leçon du 17 mars 1976 dans "Il faut défendre la société ». Cours au Collège de France, 1975-1976, Paris, Gallimard/Seuil, 1997, et le chap. « Droit de mort et pouvoir sur la vie », dans La volonté de savoir. $\rightarrow$ Voir l'article de R. Nigro.

3. Coll. « Tel»,p. 232: «La peste, celle du moins qui reste à l'état de prévision, c'est l'épreuve au cours de laquelle on peut définir idéalement l'exercice du pouvoir disciplinaire. Pour faire fonctionner selon la pure théorie les droits et les lois, les juristes se mettaient imaginairement dans l'état de nature; pour voir fonctionner les disciplines parfaites, les gouvernants rêvaient de l'état de peste. Au fond des schémas disciplinaires, l'image de la peste vaut pour toutes les confusions, et les désordres; tout comme l'image de la lèpre, du contact à trancher, est au fond des schémas d'exclusion. »

4. $N B$, p. 323. $\rightarrow$ Voir l'article de D. Sardinha.
} 


\section{Dispositifs de sécurité}

II est difficile par les temps qui courent de ne pas entendre "sécuritaire », c'est-à-dire technologies de coercition-surveillance-répression, quand le mot " sécurité » est prononcé. Or, c'est le contraire qu'il faudrait entendre dans son usage foucaldien: loin de décrire des dispositifs qui agiraient directement sur les phénomènes (hausse des prix, épidémies, conduites réprouvées) ou les personnes sous la forme d'une interdiction, d'une contrainte ou d'une discipline, Foucault désigne sous le terme de " dispositifs de sécurité " des techniques qui s'appuient sur les mécanismes du phénomène et les raisonnements spontanées des individus. II s'agit de jouer sur des variables, des éléments de contexte (STP, p. 47 et 61) qui vont induire les individus à modifier en conséquence leurs comportements, du moins au niveau des " effets de masse ». La spécificité des dispositifs de sécurité est ainsi qu'ils ne visent pas tant les corps individuels que des populations, la multiplicité des hommes comme affectée de processus d'ensemble, pour les réguler, en contrôler les effets. Foucault y voit une manière pour le pouvoir de prendre la vie en charge (naissance, mort, maladie, accident), d'où le terme de biopouvoir. Différents champs apparaissent comme les lieux d'émergence de ces dispositifs: la régulation de la population (taux de naissance, de fécondité, de mortalité, de morbidité), la mise en place d'une sécurité sanitaire (c'est-à-dire la tentative d'organiser un milieu sain, comme par exemple l'instauration d'une hygiène publique), de garanties, pour pallier les aléas de la vie (accident, maladie, vieillesse...), de technologies d'assurances individuelles et collectives, et de la protection contre le danger ${ }^{5}$.

Dans Sécurité, territoire, population, l'exemple que prend Foucault dans le champ de la «santé publique » est celui des mesures prophylactiques de variolisation-vaccination de la population face à l'épidémie de variole (p. 12 sq. et p. 59 sqq.) Il s'agit par ce procédé non pas d'écar-

5. "Il faut défendre la société », op. cit., p. 215-223. 
ter la maladie, de prévenir toute exposition à celle-ci, mais au contraire de se mettre en contact avec elle, et d'utiliser les processus biologiques spontanés - ici les réactions immunitaires - contre l'épidémie. Foucault fait ici un usage presque métaphorique de la variolisation - lui-même qualifie ce rapprochement d'analogique, mais il semble considérer qu'il y a une certaine continuité entre cette stratégie médicale reposant sur des réactions biologiques et le fait, pour obtenir les résultats collectifs espérés, de s'appuyer sur des processus et des comportements « naturels», « spontanés » - en tout cas non directement contraints - qui ont cours dans une population.

Il nous semble que l'inoculation en soi fournit tout au plus une heureuse analogie. Ce qui constitue une technique de pouvoir, certes d'un type nouveau, c'est le fait d'appliquer la vaccination à toute une population, après des calculs statistiques du rapport bénéfices-risques, la détermination de taux plus «normaux » que les autres, c'est-à-dire plus favorables, et par suite de populations et de sous-populations plus ou moins exposées à l'épidémie. Il est à noter que le procédé implique non pas de séparer les malades des non-malades, mais d'intégrer le risque dans la réaction face à l'épidémie et de s'appuyer sur lui.

L'épidémie de sida a été l'occasion du développement non pas d'un mais d'une série de dispositifs de ce type, qui ne se laissent pas enfermer dans la métaphore biologique. Il s'agit en effet de dispositifs

- qui sont dans un rapport à des taux qu'il s'agit de faire baisser - en l'occurrence, le nombre de nouvelles contaminations, puisqu'on ne guérit pas de l'infection: selon l'âge, le sexe, la nationalité, la voie de transmission, le stade d'évolution de l'infection au moment du dépistage (primo-infection, pathologies associées, stade sida...);

- par suite, qui ne portent pas tant sur des individus identifiés et individualisés que sur la population, sur des sous-populations (voir STP, p. 62) dans lesquelles on observe une prévalence supérieure à la moyenne;

- qui n'ont pas tant pour fonction d'interdire des pratiques ou de les contrôler que de s'appuyer sur elles pour y introduire les éléments nécessaires de prévention $\left(S T P\right.$, p. $\left.39^{6}\right)$;

6. Voir notamment l'exemple de la réduction des risques pour les usagers de drogues par voie intraveineuse: dans le cadre de la lutte contre le sida, il s'agissait non pas de les punir ou de les sevrer, mais d'éviter d'abord qu'ils ne s'infectent. C'est le rôle de la mise à disposition de seringues stériles - qui 
- qui ne procèdent pas par séparation des malades et des non-malades, exclusion des malades, des personnes contaminées ou considérées comme «à risque ». Les campagnes de prévention indiquent des procédures qui s'appliquent à tout le monde, séropositifs comme séronégatifs (ou personnes ignorant leur sérologie), pour éviter contamination et surcontamination - cependant une tension se fait jour entre la distinction de sous-populations et l'indifférenciation des procédures;

- qui sont destinés non pas seulement à réagir à des événements ou des actes effectifs, mais qui visent des événements potentiels, qui s'adressent à des risques, à un « danger» (STP, p. 63).

Par certains aspects, cela pourrait relever du modèle disciplinaire, puisqu'il s'agit de modifier les comportements individuels. Les associations ont ainsi pu être accusées d'être les agents d'imposition de nouvelles normes morales sexuelles. Cependant, cela n'est assorti d'aucun contrôle ni surveillance, et il s'agit plutôt de faire entrer les gestes de protection dans les mœurs collectives à travers des campagnes publiques. Celles-ci s'adressant à des individus responsables, elles proposent plus une conduite, un gouvernement de soi, qu'elles n'imposent une discipline.

Il ne faut pas lire l'émergence de ces dispositifs non contraignants comme le progrès naturel et irréversible vers la raison et la liberté. Le choix de ces techniques n'a pas été opéré dans l'unanimité, il a fait l'objet de multiples débats et controverses; il est issu des rapports de forces et des positionnements des différents acteurs intervenus sur la question. Foucault souligne qu'il n'y a pas simple succession dans le temps des différentes technologies de pouvoir; il y a des dominantes ou des corrélations qui changent, et une histoire des technologies en général montrerait « comment une dominante va faire jouer des éléments d'autres ordres dans sa tactique propre» (STP, p. 10). Il aurait pu y avoir addition ou plutôt potentialisation mutuelle des différentes techniques de pouvoir, à la façon de ce que Foucault décrit dans Surveiller et Punir: "C'est le propre du XIX ${ }^{\mathrm{e}}$ siècle d'avoir appliqué à l'espace de l'exclusion dont le lépreux était l'habitant symbolique (et les mendiants, les vagabonds, les fous, les violents formaient la population réelle) la technique de pouvoir propre au quadrillage disciplinaire.» (p. 232.)

dépend en dernière instance de leur emploi par les usagers eux-mêmes. Le nombre de nouvelles contaminations chez ceux-ci a effectivement chuté. 
Ce qu'il résume de la formule: «Traiter les "lépreux" comme des “pestiférés". » À ces deux techniques se serait ajoutée la saisie statistique du risque, faisant entrer dans l'espace d'exclusion-surveillance les souspopulations à forte prévalence.

L'un des éléments centraux dans ces agencements des techniques du pouvoir est la modalité d'appréhension du risque, selon qu'il est saisi comme danger ou probabilité. Tandis que les normes jurico-légales tendaient à exclure un danger, à l'écarter, et les technologies disciplinaires à le contenir, à le maîtriser, comme quelque chose d'étranger, une figure de l'ennemi, tantôt extérieur, tantôt intérieur, les dispositifs de sécurité impliquent de s'approprier le risque, de l'intégrer dans ses choix, dans un calcul collectif et individuel. L'appréhension de l'épidémie et de la contamination va osciller entre danger-ennemi et risque réapproprié. Cette tension apparaît nettement dans la traduction des savoirs statistiques eux-mêmes en dispositifs pratiques de lutte contre l'épidémie.

Les statistiques et leur usage au sein d'instances de vigilance épidémiologique sont au cœur de l'histoire de l'épidémie. La nouvelle maladie est identifiée à partir de cas, d'une série de $\operatorname{cas}^{7}$ de sarcomes de Kaposi (type relativement rare de cancer de la peau) présentés par des hommes qui sortent des statistiques épidémiologiques habituelles, selon des critères d'âge et d'origine ethnique, mais qui présentent la caractéristique commune d'être homosexuels ${ }^{8}$. La découverte d'une prévalence dans une population donnée prend une valeur heuristique, pour identifier de nouveaux cas ainsi que pour formuler des hypothèses quant aux modes de transmission: par voies sexuelles et sanguines ${ }^{9}$, avant même que soient identifiés de manière certaine les vecteurs de contamination.

Mais ces catégories qui se révèlent avoir une pertinence statistique dans l'approche de cette maladie ne sont pas neutres. Elles véhiculent nombre de représentations sociales chargées de préjugés, de vieilles peurs. Elles ont des incidences jusque dans la formation du savoir scientifique. Des cas qui présentent des symptômes typiques communs mais qui n'appartiennent pas aux premiers groupes sociaux identifiés sont ainsi ignorés. Et lorsqu'un troisième groupe à forte prévalence est identifié, qui a pour caractéristique commune non les pratiques (homo-

7. Sur la notion de cas, voir STP, p. 62.

8. Mirko Grmek, Histoire du sida, Paris, Payot, 1995, p. 31 sq.

9. En s'appuyant sur le modèle de l'hépatite B, quand on s'aperçoit que l'épidémie touche également les usagers de drogue par voie intraveineuse. 
sexualité, toxicomanie) mais l'origine, Haïti, l'hypothèse qui prévaut alors est que l'épidémie a été amenée aux États-Unis par des Haïtiens ${ }^{10}$. Par la suite, avec l'identification du virus et de ses différentes souches et mutations, on retiendra plutôt l'hypothèse inverse: l'épidémie aurait probablement été amenée en Haïti par des touristes américains.

Les enjeux de la traduction de la prévalence de l'épidémie dans certains groupes apparaissent ainsi dès les début de l'épidémie: c'est le petit nombre de cas et le fait qu'elle touche des groupes précis qui a été mis en avant dans les premiers temps - cela afin d'éviter tout affolement de la population ( " générale »), mais c'était aussi une manière d' " externaliser» la maladie en la réservant à des groupes de «déviants» ou d'étrangers, ou en les percevant comme étant eux-mêmes source de danger. C'est ainsi que la notion de " groupe à risque » est forgée - ce qu'on a appelé les quatre $\mathrm{H}$, notamment dans les médias: Homosexuals, Heroinomans, Haïtians, Hemophils, auquel un cinquième pouvait être ajouté, Hores (prostituées).

Cette notion tend à faire d'une catégorie statistique une identité figée. De plus, elle néglige cet élément dont Foucault montre qu'il est pris en compte par les dispositifs de sécurité: la circulation (STP, p. 16 sq.), comme s'il n'y avait aucun commerce entre les différents groupes. C'est au moment où on s'est aperçu que l'épidémie n'avait pas respecté les frontières des dits «groupes à risque» que les pouvoirs publics ont commencé à agir.

La question de savoir si la prévalence chez les homosexuels devait être mise en avant, pour diffuser un message de prévention ciblé, ou tue, pour éviter le renforcement de stigmatisations existantes ou l'idée que l'épidémie serait cantonnée à ce groupe, a fait l'objet de violents débats. Mais la présence importante d'homosexuels au sein des associations qui les premières étaient sur le terrain a permis d'emblée de poser les questions à partir d'un «nous », qui n'était pas le «nous » exclusif de la majorité hétérosexuelle.

L'existence d'un mouvement associatif actif, le fait que de nombreux militants étaient issus de ces groupes désignés comme « à risque » et touchés de manière plus visible par l'épidémie, mais aussi que l'intérêt des malades et des personnes touchées a été mis au centre de leur action,

10. Mirko Grmek, op. cit., p. 73 sqq. 
tout cela a joué un rôle central dans l'orientation et la mise en place des dispositifs de lutte contre le sida.

La place des associations dans ces dispositifs, leur relation aux médecins et aux pouvoirs publics ne sont cependant ni univoques ni monolithiques. C'est l'analyse du pouvoir que propose Foucault dans La volonté de savoir qu'il faut ici convoquer (p. 121 sqq.). Car ces associations ont pu apparaitre comme une force de résistance, face tout d'abord à l'inertie des pouvoirs publics, puis contre une volonté d'imposer des mesures coercitives, face également au pouvoir médical, et contre des normes sociales et morales stigmatisantes et excluantes; mais réduire les enjeux de pouvoir à ces résistances serait se méprendre sur leurs configurations multiples et mouvantes. Les associations ne forment pas un tout homogène, elles peuvent entrer en tension entre elles sur des revendications et des stratégies et se trouver dans des enjeux de domination de l'espace associatif. Des médecins peuvent y participer ou travailler avec elles. Et elles ne sont pas uniquement en position de résistance par rapport à un pouvoir étatique normatif et répressif: se trouvant en première ligne dans la diffusion de l'information et dans les campagnes de prévention, elles sont aujourd'hui le relais d'une partie des politiques publiques, et reçoivent pour cela un financement public.

Nous terminerons par deux remarques qui portent toutes deux sur la relation entre les individus et la population au sein des dispositifs de sécurité et de la lutte contre le sida.

Foucault désigne comme cible du pouvoir agencé selon les « dispositifs de sécurité » non pas le sujet doué d'une volonté qui se soumet, qui obéit à la volonté d'une autre volonté, souveraine, ni le corps objet d'une discipline, éduqué, surveillé, rééduqué, mais les comportements collectifs d'une population, comportements qui sont censés s'ajuster sur des éléments de contexte sur lesquels on joue.

L'individu supposé derrière le membre de la population sur le «bon » comportement duquel on compte en dernière instance (STP, p. 45), c'est l'individu capable de calculs, de prévisions et de calculs rationnels, d'un mode de raisonnement prévisible sur lequel le gouvernement cale son action. Mais, d'une part, cela suppose un individu qui agisse à partir d'une évaluation de type bénéfice-risque; d'autre part, cela suppose de savoir comment il procède à cette évaluation: va-t-il évaluer le risque qu'il court à partir de son mode de vie, à partir de celui qu'il attribue à ses partenaires? Est-ce que le risque d'attraper une maladie grave et à 
terme mortelle justifiera à ses yeux la nécessité d'une protection systématique?

Le « calcul rationnel » se heurte en fait à un imaginaire tissé de représentations.

L'épidémie de sida aurait ainsi pu faire exploser de grandes hypocrisies autour de la sexualité et du couple: l'affirmation de la monogamie des couples hétérosexuels, la stigmatisation des femmes multipartenaires, le non-dit autour du multipartenariat des hommes. Mais silence, invisibilité et déni de l'épidémie ont prévalu dans la population hétérosexuelle.

De plus, on observe la persistance de pratiques «magiques» de prévention (évaluation du risque couru avec une personne à sa «bonne tête », à son milieu social, au désir ou à l'amour qu'on éprouve pour elle; pratiques du retrait avant éjaculation, lavage à l'eau de javel d'objets touchés par une personne séropositive), même chez des personnes censées avoir reçu une bonne information.

Il s'agit là non pas tant des « contre-conduites » décrites par Foucault que de résistances externes (STP, p. 197 sq.). Ce qui pourrait constituer des contre-conduites serait plutôt le développement d'autres modèles: contre le sexe/l'amour sans risque, le «bareback», (au sens propre monter « à cru », littéralement « cul nu »), idéologie qui prône la prise de risque et le sexe non protégé, ou au contraire, la fidélité ou l'abstinence.

Cela nous conduit à la seconde remarque, sur la notion de «nature » au sein du « biopouvoir ». Elle apparaît ambivalente ou tout au moins plurivoque. Foucault dit des dispositifs de sécurité qu'ils donnent une place à la vie dans le groupe politique, qu'ils visent l'homme « en tant qu'espèce » $(S T P$, p. $23 s q)$ : les processus naturels - vie, mort, maladie, vieillesse - y sont pris en compte. Mais il ne s'agit pas tant ici de l'espèce que de la population, définie par une société. Et dans la notion de population, Foucault ne s'intéresse pas seulement aux processus vitaux, biologiques qui y ont cours. Ce n'est pas non plus la question du peuplement, le nombre d'habitants et leur répartition sur le territoire, qui l'occupe ici. Il s'intéresse à la manière dont le pouvoir peut prendre en compte la manière dont agissent «naturellement » les membres de la population, naturellement signifiant ici «spontanément» $(S T P$, p. 359), telle qu'on peut en dégager des mouvements de masse, en déduire des lois, et par suite s'appuyer sur ces lois pour obtenir un résultat souhaité. 
Foucault joue sur ces différents sens de nature, et s'il dit bien qu'il ne s'agit pas d'une «prime nature», il parle de «nature sociale». Cependant, si les comportements sociaux répondent à certaines logiques, ces logiques ont une histoire et n'existent pas en soi, pas plus qu'elles ne sont réductibles à des calculs d'intérêt. Foucault dégage cependant un certain type de technologie de pouvoir qui agit en donnant une place à ces conduites qui échappent en partie aux tentatives volontaristes d'y intervenir directement.

Les dispositifs de lutte contre le sida jouent sur un calcul fait par les individus, mais ils vont au-delà: ils en appellent à leur responsabilité, et entendent s'appuyer sur l'information pour développer chez eux une plus grande capacité d'agir. Plus encore, ce qui est apparu, c'est que le développement de cette capacité d'agir, d'adopter des conduites de protection pertinentes, est indissociable des mouvements collectifs qui la portent. La force des mouvements associatifs dans les débuts de l'épidémie a fortement déterminé tant l'agencement que l'efficacité des dispositifs de pouvoir: refus d'une stigmatisation des malades et des dispositifs contraignants, exigence de campagnes de prévention adaptées, intégration des pratiques de protection, notamment par les homosexuels, les prostituées et les toxicomanes, déplaçant les représentations qui les entourent. Mais les campagnes d'information, sans doute insuffisantes, ont révélé leurs limites. Là où ces mouvements n'ont pas eu lieu ou apparaissaient comme extérieurs, les pratiques de protection peinent à s'imposer, comme dans la population hétérosexuelle, ou peuvent être délaissées, comme aujourd'hui avec ce que l'on a appelé le relapse (relâchement des pratiques de protection) dans le milieu gay, tandis que l'arrivée des traitements a induit une moindre visibilité de l'épidémie (retard de l'entrée en phase sida, diminution du nombre de décès) et une baisse de la mobilisation militante. 